
\title{
SB Research S Suare
}

\section{Experiences of Using Hybrid Operating Rooms in the Treatment of large or giant Carotid-Ophthalmic Aneurysms}

Nai Zhang

Tianjin Medical University General Hospital

Wen-qiang Xin ( $\nabla$ xinwenqiangdr@126.com )

Tianjin Medical University General Hospital

\section{Research article}

Keywords: balloon, microsurgical clipping, hybrid operating room, large or giant carotid-ophthalmic aneurysm

Posted Date: December 30th, 2019

DOI: https://doi.org/10.21203/rs.2.19707/v1

License: @ (i) This work is licensed under a Creative Commons Attribution 4.0 International License. Read Full License 


\section{Abstract}

Background: A hybrid operating room (Hybrid-OR) was a surgical theatre combined conventional operating room with advanced medical imaging devices. There were still plenty of limitations when endovascular treatment or microsurgical treatment was used individually to deal with large or giant carotidophthalmic aneurysms. The purpose of this study was to explore and summarize the technical features and effectiveness of the application of Hybrid-OR in dealing with major intracranial carotid-ophthalmic aneurysms.

Methods: The department of neurosurgery treated 12 cases of large or giant intracranial carotid-ophthalmic aneurysms between March 2013 and December 2015 with Hybrid-OR. All cases were treated with clipping and parent vessel reconstruction.

Results: With the assistance of Hybrid-OR, the rate of incompletion of intraoperative aneurysm clipping decreased from the original $25 \%$ ( $3 / 12$ ) to $0 \%$, while the rate of vessel stenosis decreased from the original $16.7 \%(2 / 12)$ to $8.35 \%(1 / 12)$. For thromboembolic event, there was one ischemic infarction complication occurred in only one patient, and none of patients appeared embolic infarction complication. All 12 patients were followed up for an average of 3 years and no aneurysms recurred. The postoperative recovery conditions were evaluated with the modified Rankin Scale (mRS): 11 patients showed no symptoms (mRS 0), 1 patient showed slight disability (mRS 1-2), and none of the cases showed severe disability (mRS 5) nor death (mRS 6).

Conclusion: The Hybrid-OR provides new ideas to the surgical clipping the large or giant intracranial carotid-ophthalmic aneurysms, which effectively decreased the rate of intraoperative vessel stenosis and unsuccessful clippings.

\section{Background}

Intracranial carotid-ophthalmic aneurysm referred to the aneurysm that originates from the distal dural ring of the internal carotid artery (ICA) at the top of the cavernous sinus to the origin of the posterior communicating artery (PComA). It was known as a C6 aneurysm or para-clinoid aneurysm which accounted for about $5 \%$ of intracranial aneurysms.[1,2] The rates of fatality and disability were relatively high since its location of growth, complexity and specificity of ophthalmic segment aneurysm.[1, 2] Although endovascular and micro-neurosurgical technologies have been developing rapidly, ophthalmic segment aneurysms, especially for the large (diameter $=10-25 \mathrm{~mm}$ ) and giant (diameter $>25 \mathrm{~mm}$ ) ones, were still considered as a challenge for surgeons.

Hybrid operating room (Hybrid-OR) was known as the "one-stop" operating room. In a broad sense, it indicated that one operating room was equipped with multiple medical examination devices and operation assisting devices. For instance, intraoperative MRI and navigation devices, and other treatment equipment was included so that imagological examinations, tracking, navigation assistance and multiple surgical approaches could be combined to perform in one operating room. In a narrow sense, Hybrid-OR indicated that endovascular-surgical combined operating rooms not only retain the advantages of traditional open operations (visibility and convenience for operating and navigating), but also kept the advantages of endovascular operations (less trauma and multiple endovascular assisting approaches).

Hybrid operation was originally defined by Angelina et al.[3] in 1996, and firstly used for the cardiac operation. Recently, the hybrid operation has been providing new ideas for neurosurgeons in treating cerebrovascular diseases. Our hospital completed the building of Hybrid-OR in 2011, which effectively treated 12 intracranial large and giant carotid-ophthalmic aneurysm cases between March 2012 and December 2015, and its effectiveness was summarized in our study.

\section{Methods}

\section{Clinical data and Imageology data}

During the period from March 2013 - December 2015, our department of neurosurgery applied Hybrid-OR in a treating large and giant carotid-ophthalmic aneurysm in 12 cases: 5 male cases and 7 female cases. The ages of these patients ranged from 53 to 68 years old with a mean of 53.9 years. All cases showed unruptured intracranial aneurysms, including 5 cases of chronic headache, 4 cases of visual impairment, and 3 cases of visual field defects.

All cases were diagnosed with computed tomography angiography (CTA), magnetic resonance imaging (MRI), preoperative digital subtraction angiography (DSA) and 3D-DSA examinations. And the ICA balloon occlusion test (BOT), cross circulation test and Allcock's test were performed to understand the condition of cerebral blood flow compensation. 12 patients were diagnosed with 12 large or giant aneurysms. The maximum diameter of the aneurysmal dome varied from 10.5 to $38.1 \mathrm{~mm}$, the neck measured from 3.9 to $15.4 \mathrm{~mm}$ and the dome-neck $(\mathrm{D} / \mathrm{N})$ ratio varied from 1.4 to 9.8 . Summary of demographic and clinical characteristics of 12 patients are shown in Table 1. 
Table 1

Summary of demographic and clinical characteristics of 12 patients

\begin{tabular}{|c|c|c|c|c|c|c|c|c|c|c|c|}
\hline No. & Age/sex & Side & $\begin{array}{l}\text { Maximal } \\
\text { diameter(mm) }\end{array}$ & $\begin{array}{l}\text { Neck } \\
\text { size }(\mathrm{mm})\end{array}$ & $\begin{array}{l}\mathrm{D} / \mathrm{N} \\
\text { ratio }\end{array}$ & $\begin{array}{l}\text { Balloon } \\
\text { location } \\
\text { in } \\
\text { operation }\end{array}$ & $\begin{array}{l}\text { Residual } \\
\text { aneurysm } \\
\text { neck was } \\
\text { found by } \\
\text { intraoperative } \\
\text { DSA }\end{array}$ & $\begin{array}{l}\text { Vessel } \\
\text { stenosis was } \\
\text { found by } \\
\text { intraoperative } \\
\text { DSA }\end{array}$ & $\begin{array}{l}\text { Times of } \\
\text { inter- } \\
\text { operation } \\
\text { DSA }\end{array}$ & $\begin{array}{l}\text { Thromboembolic } \\
\text { event }\end{array}$ & $\begin{array}{l}\mathrm{mRS} \text { at } \\
\text { discharge }\end{array}$ \\
\hline 1 & $56 / F$ & $\mathrm{R}$ & 25.3 & 4.5 & 5.6 & $\begin{array}{l}\text { C2 - C3 of } \\
\text { ICA }\end{array}$ & + & $\begin{array}{l}\text { Ophthalmic } \\
\text { artery \& ICA } \\
\text { stenosis }\end{array}$ & 3 & - & 0 \\
\hline 2 & $54 / F$ & $\mathrm{R}$ & 21.4 & 13.4 & 1.6 & $\begin{array}{l}\text { Aneurysm } \\
\text { neck }\end{array}$ & + & ICA stenosis & 3 & - & 0 \\
\hline 3 & $35 / F$ & $\mathrm{R}$ & 13.7 & 9.5. & 1.4 & $\begin{array}{l}\text { Aneurysm } \\
\text { neck }\end{array}$ & - & - & 1 & - & 0 \\
\hline 4 & $45 / \mathrm{F}$ & L & 17.1 & 6.5 & 2.6 & $\begin{array}{l}\text { C2 - C3 of } \\
\text { ICA }\end{array}$ & - & - & 1 & - & 0 \\
\hline 5 & $64 / F$ & $\mathrm{R}$ & 23.8 & 9.3 & 3.9 & $\begin{array}{l}\text { Aneurysm } \\
\text { neck }\end{array}$ & - & - & 1 & - & 0 \\
\hline 6 & $56 / \mathrm{F}$ & $\mathrm{L}$ & 25.9 & 7.1 & 2.6 & $\begin{array}{l}\text { C2 - C3 of } \\
\text { ICA }\end{array}$ & - & - & 1 & $\begin{array}{l}\text { Ischemic } \\
\text { infarction in } \\
\text { operation }\end{array}$ & $1-2$ \\
\hline 7 & $62 / \mathrm{F}$ & $\mathrm{R}$ & 26.8 & 15.4 & 1.7 & $\begin{array}{l}\text { Aneurysm } \\
\text { neck }\end{array}$ & + & - & 2 & - & 0 \\
\hline 8 & $69 / F$ & L & 13.2 & 4.7 & 2.8 & $\begin{array}{l}\text { C2 - C3 of } \\
\text { ICA }\end{array}$ & - & - & 1 & - & 0 \\
\hline 9 & $67 / F$ & $\mathrm{R}$ & 10.5 & 5.9 & 1.8 & $\begin{array}{l}\text { C2 - C3 of } \\
\text { ICA }\end{array}$ & - & - & 1 & - & 0 \\
\hline 10 & $45 / \mathrm{F}$ & $\mathrm{R}$ & 38.1 & 3.9 & 9.8 & $\begin{array}{l}\text { C2 - C3 of } \\
\text { ICA }\end{array}$ & - & - & 1 & - & 0 \\
\hline 11 & $43 / \mathrm{M}$ & L & 14.1 & 4.8 & 2.9 & $\begin{array}{l}\text { C2 - C3 of } \\
\text { ICA }\end{array}$ & - & - & 1 & - & 0 \\
\hline 12 & $59 / F$ & L & 33.0 & 8.3 & 3.5 & $\begin{array}{l}\text { Aneurysm } \\
\text { neck }\end{array}$ & - & - & 1 & - & 0 \\
\hline
\end{tabular}

Note: $\mathrm{DSA}=$ digital subtraction angiography; ICA = internal carotid artery; D/N = dome-neck; $\mathrm{mRS}=$ Modified Rankin Scale.

\section{Operation methods and technology analysis}

First, preoperative Hybrid-OR preparation: all patients were placed in the supine position after general anesthesia. Unilateral femoral puncture by Seldinger technique, then a 6-F guiding catheter was placed in the C1 of ICA of the ipsilateral side of the aneurysm with continuous flushing with 5,000 U heparin per liter normal saline solution. MTI balloon(Hyperglide, EV3/4 mm x 20mm)deployment: when the neck of aneurysm $\geq 8 \mathrm{~mm}$, the balloon was deployed across the neck of the aneurysm; when the neck of aneurysm $<8 \mathrm{~mm}$, the balloon was deployed in C2 - C3 of ICA. Based on this principle: the balloon was placed in the C2 - C3 segment of ICA for 7 cases (1, 4, 6, and 8-11) and across the aneurysm neck for 5 cases $(2,3,5,7$ and 12), and the balloons kept deflated after initial deployment. Neither pre-procedural nor intra-procedural antiplatelet, systemic heparinization agents were administered.

Second, the head was secured by a carbon fiber fixation system, and the electrophysiological monitoring was used. Conventional pterional approach: using the intradural anterior clitoridectomy (IAC) technique to remove the anterior clinoid process (ACP). The distal dural ring (DDR) of the optic-sheath was opened to sufficiently dissociate the nerve from the aneurysm neck.

Third, the balloon would be inflated to block the ICA when clipping the aneurysm. After the aneurysms clipped, intraoperative DSA was administered repeatedly to identify residues of the aneurysms neck and vessel stenosis because of incorrect clipping. If the clipping was not ideal, then the clip would be readjusted to achieve the best effect.

\section{Results}

Residual aneurysm necks were discovered by the intraoperative DSA in 3 cases (1, 2 and 7): for these 3 cases, the residual aneurysm necks were successfully clipped by adjusting the clips, that were verified by the second intraoperative DSA. With the assistance of Hybrid-OR, the rates of the residual aneurysm necks decreased from the original $25 \%(3 / 12)$ to $0 \%$. 
Vessel stenosis were found in 2 cases (1 and 2 ) by the intraoperative DSA: for case 1, clipping was readjusted, and second intraoperative DSA displayed that the stenosis of the parent carotid artery and ophthalmic artery was relieved; while for case 2, clipping was readjusted, but mild stenosis of the parent ICA still remained as severe atherosclerosis in the aneurysm neck. The intraoperative electrophysiology monitor showed no abnormalities. With the assistance of Hybrid-OR, the rate of vessel stenosis decreased from the original $16.7 \%(2 / 12)$ to $8.35 \%(1 / 12)$.

For thromboembolic event, there was one patient occurred ischemic infarction complication (case 6), none of patients appeared embolic infarction complication. She underwent contralateral limb weakness after the operation. Her pre-operation BOT demonstrated clinical intolerance (positive), and preoperation cross circulation test demonstrated inadequate compensation. The balloon was inflated to block the ICA in the C2 - C3 segment of ICA for 45 minutes, and hyper-intensity signals located at Watershed area of operating side hemisphere was found in the DWI of post-operation MRI images. It was considered the ischemic infarction was caused by the balloon block and inadequate compensation.

The postoperative conditions were evaluated with the modified Rankin Scale (mRS): 11 patients showed no symptoms (mRS = 0), 1 patient showed slight disability (mRS $=1-2)$, and none patients showed severe disability $(m R S=5)$ nor death $(m R S=6)$. All patients were followed up for an average of 3 years. Postoperative CTA was performed to observe the effect of occlusion of the aneurysms,and no aneurysms recurred. The more details of patients were shown in Table 1.

\section{Illustrative Cases:}

\section{CASE1: Female patient, 56 years old}

A 56-year-old female presented to our institution with progressive vision loss. Her medical history was noncontributory. An ophthalmological evaluation showed a right central scotoma. Cranial MRI showed a mass suggestive of a carotid artery aneurysm. DSA revealed a $25.3 \times 18.5 \mathrm{~mm}$ aneurysm with a $4.5 \mathrm{~mm}$ neck arising from the medial wall of the right ophthalmic segment (Fig. 1A\&B), and the BOT of ICA and Allcock's test were performed to understand the condition of cerebral blood flow compensation. To clip the aneurysm and reconstruct the right ICA, she was offered both surgical and endovascular treatment, along with alternatives, risks, and benefits. The management plan was formulated to proceed with the hybrid operation as an elective procedure. In the operation, we put the balloon was placed in the C2 - C3 segment of ICA due to a relatively narrow-necked aneurysm (D/N ratio 5.6). Then we did craniotomy by taking pterional approach: using the IAC technique to remove the ACP. Then we found the right optic nerve was compressed by the giant aneurysm and the optic nerve is crossed by the aneurysm neck; removal of the anterior clinoid process and dissociation of the optic nerve from the aneurysm neck to sufficiently reveal the aneurysm neck (Fig. 1C\&D). After the placement of the first clip, the first intraoperative DSA displayed that the aneurysm had been clipped and small amounts of the residue of the aneurysm neck (Fig. 1E\&F). A supplementary miniature clip was placed during the operation. The second intraoperative DSA displayed that the aneurysm had been completely clipped. However, stenosis occurred in the ICA (white arrow) with poor visualization of the optic artery (black arrow) and poor visualization of the distal branch vessel of ICA (Fig. 1G\&H). A miniature clip with angular tips was used to replace the original one. The third intraoperative DSA displayed that the aneurysm was completely clipped. The ICA with stenosis had been relieved (white arrow) and the visualization of the optic artery and distal branch vessel of MCA was effectively improved (black arrow) (Fig. 1I\&J).

\section{CASE2: Female patient, 54 years old}

A 54-years-old woman presented with a 6-month history of chronic headache. MRI and pre-operation DSA provided the diagnosis of a 21.4-mm large carotidophthalmic aneurysm with a 13.4-mm wide neck. (Fig. 2A\&B) A combined approach was offered in the Hybrid-OR. Firstly, a $4 \mathrm{~mm} \times 20 \mathrm{~mm}$ balloon (Hyperglide; Micro Therapeutics, Inc., Irvine, CA) was deployed across the ophthalmic aneurysm neck, and the balloon was inflated (Fig. 2C\&D). The aneurysm softened sufficiently to place two arrays of straight fenestrated and angled fenestrated clips (Aesculap AG, Tuttlingen) on the aneurysm. After that, the second intraoperative DSA was performed and found the bulk of aneurysm was clipped and not visualized, but a small portion of the aneurysm was residue and visualized (Fig. 2E\&F). After adjusting clips, the third intraoperative DSA displayed the aneurysm was completely clipped, but the parent artery was slightly narrowed due to the severe calcification of the aneurysmal neck (Fig. 2G\&H).

\section{Discussion}

\section{Limitations of Clipping Large or Giant Carotid-Ophthalmic Aneurysms by Using Conventional Microsurgery}

The size and shape of the aneurysms were the key issues for such operations. Previous studies reported that the rates of fatality and morbidity were still as high as $20-30 \%$ in neurosurgical centers which had well-developed technologies, despite that patients could take safer and relatively simpler operations such as aneurysm exclusion and EC-IC bypass. The critical difficulties of performing these operations are the proximal control of the ICA; the field of vision influenced by the aneurysm, width of the aneurysm necks, complex branches and perforating blood vessels, formation of endoluminal thrombosis, and as well as atherosclerosis and dysplasia. For the clipping of giant carotid-ophthalmic aneurysm, control of the proximal end of the parent artery was the most crucial step. In treating some patients with giant carotid-ophthalmic aneurysms, the removal of ACP would provide sufficient space between the aneurysm neck and the DDR of cavernous sinus for the placement of temporary blocking clips, including control of the proximal end of the ICA.

The vast majority of patients with giant carotid-ophthalmic aneurysms required an incision of neck to be performed first to do proximal ICA control. However, both ACP removal and neck incision would increase operational trauma and lengthen surgical time, especially for elderly patients over the age of 65 . Elderly

Page $4 / 9$ 
patients were less tolerant for trauma. The research by ISAT subgroup presents that the patients over the age of 65 have higher rates of epilepsy, lung infection, cerebral vasospasm, infarction and neurological complications.[4] Therefore, intraoperative neck incision and proximal part blocking should be prevented in treating carotid-ophthalmic aneurysm patients. This should minimize surgical trauma and shorten the operation time.

Due to the size of the carotid-ophthalmic aneurysms, the aneurysm necks located in ICA leading to the skull, around the ACP, and the optic nerve blocked the field of vision. As a result, finding the aneurysm neck and its branches through craniotomy would be difficult due to blind spots. Especially large and complex intracranial aneurysms, the aneurysm neck might be obstructed which could lead to it rupturing before clipping.[5] As reported in the literature, after conventional aneurysm clipping with intraoperative indocyanine green (ICG) video angiography check, post-operation DSA showed the rate of incompletion of aneurysm clippings was at $6.3 \%$ while parent vessel stenosis was $5.7 \%$. For giant aneurysms, the rates of incompletion of clippings and occurrence of stenosis were as high as $15 \%$ even more.[6] So, compared to intraoperative DSA, intraoperative ICG could not demonstrate clearly and accurately images of parent vessel stenosis and aneurysm neck residues due to blocked vision and other reasons. Besides, intraoperative ICG was not able to directly observe the situation of the distal blood vessels.

\section{Limitations of Intra-Arterial Embolization for Treating Giant Carotid-Ophthalmic Aneurysms}

Recently, interventional treatments for intracranial aneurysms have been in rapid development, which could reveal its advantages in treating intracranial aneurysms. Currently, usable interventional technologies in treating complex wide-necked aneurysms are balloon-assisted coiling embolization, stent-assisted embolization and dual micro-catheter coiling embolization. However, a wide-necked aneurysm with complex conformation was difficult to be totally removed by endovascular treatments. Although new interventional devices, tools, and technologies are evolving, a variety of interventional techniques are techniques challenging for treating giant carotid-ophthalmic aneurysm. For the method of stent-assisted coiling embolization, it is possible that the stent guidewire could not overcome the technical difficulties of placing the stent through an aneurysm. The stent is used in stent-assisted coiling embolization to reconstruct the parent vessel and set as a block on the aneurysm neck and to prevent coil dislocation. However, within a few months of surgery, dual antiplatelet therapy (DAPT) must be strictly performed to the patients until the internal membrane of the parent vessel has completely regenerated before the aneurysm neck.[7] Therefore, in most cases, stent-assisted coiling embolization technology could not be safely used to treat ruptured aneurysms.[8]

Carotid-ophthalmic aneurysms often show mild symptoms or none until they grow to a larger size due to their unique anatomic features. It was reported $30-$ $50 \%$ of patients who have had carotid-ophthalmic aneurysms grown to giant size (diameter $>25 \mathrm{~mm}$ ) on their first visit to the hospital. The main symptoms are often loss of vision and visual field defects.[9] And intervention embolization was used to treat these patients; however, the aftermath of the aneurysm could not be eliminated, and the symptoms were not only not alleviated after the operation, but also keep growing progressively which requires craniotomy to remove emboli in order to save the patient's' vision and visual field[10,11]. Moreover, carotid-ophthalmic aneurysms often contain important vessels or artery branches which originates from the aneurysm dome or fundus parts such as the ophthalmic artery, PComA, and even anterior choroidal artery (AChA).

Under these circumstances, the ideal treatments were to reconstruct the parent vessel based on retaining important perforator vessels and its branches. In this case, using the common interventional embolization technologies and flow-directed devices to solve the aforementioned problem was pretty difficult. Furthermore, another difficulty in treating giant complex carotid-ophthalmic aneurysms was the high risk of relapse after surgery. As reported, the incidences of relapse after carotid-ophthalmic aneurysm operations ranged 27.4 to $31.6 \%,[12-14]$ however, the rates of relapse after interventional embolization had reached $50 \%$ in treating giant aneurysm.[15] Therefore, It was extremely difficult to use the interventional embolization method to treat patients with widenecked, giant and/or branch vessel accumulated carotid-ophthalmic aneurysms.

In recent years, the flow diverters (e.g. SILK embolization device, Pipeline embolization device) have been widespread used to treat complex anterior circulation aneurysms, especially the giant types of the carotid-ophthalmic segment and clinoid segment. But publications reported that flow diverters used for dealing with giant anterior circulation aneurysms, dissecting aneurysms and microaneurysms were relatively rare. However, due to the rigidity and low compliance characterized by the hardness of the dense diverter, the excessive friction when the diverter went through tortuous vessel parts, for instance, the ICA siphon, might cause stent translocation, insufficient expansion, stent fracture (SF), in-stent restenosis (ISR) or occlusion, and covered branch or perforator vessel stenosis or occlusion. Besides, after placing the flow-diverter stent, the follow-up treatment would be extremely difficult if there were recurrent or residual aneurysms.

\section{The Advantages of Hybrid-OR in Dealing with Large or Giant Carotid-Ophthalmic Aneurysms}

Current surgical limitations have been overcome due to this technological breakthrough, while expanding the potential of surgery. The DSA system in the Hybrid-OR provided neurosurgeons with a brand new, intraoperative, image-guided environment, which eliminated the limitation of traditional operating rooms and DSA systems when they functioned individually.

Firstly, using balloon occlusion with the Hybrid-OR could quickly block the proximal end of the ICA. The control of the proximal end of the ICA had the following advantages: (I)preventing neck incisions, shorten operation time, and reduce surgical trauma; (II)preventing operation of two surgical sites, which reduces the possibility of complications of intracranial infection; (III) reducing operations on the ICA, which could reduce the rate of complications of intraoperative vasospasm and cerebral infarction; and (IV) dealing with ACP using the IAC technique rather than EAC technique, and no need to totally remove $A C P$, but removed a part of $A C P$ in accordance with operational needs. IAC technique was safer and more timesaving than EAC.

Page $5 / 9$ 
Secondly, compared to direct observe under the microscope or interoperation ICG technique, it's more clearly and accurately to evaluate the effect of aneurysm-clipping using the intraoperative DSA. It was extremely difficult under the microscope in 360 degrees to detect and observe the relationship between the aneurysm and their surrounding branch vessels, giant carotid-ophthalmic aneurysms were in big size, deep location, shade of ACP and optic nerve and wide necks. And it was impossible using ICG technology to observe aneurysms residual, ICA stenosis, preservation of important perforator vessels, and the status of the distal blood supply.[16] These were the reasons why the rates of incompletion of clippings and occurrence of stenosis were as high as $15 \%$ and more in conventional aneurysm clipping method with intraoperative ICG verification. Nevertheless, Hybrid-OR is applied to observe and evaluate aneurysmclipping in 360 degrees by intraoperative DSA. As shown in our cohort, 3 incomplete clipping events $(1,2$ and 7$)$ and 2 vessel stenosis events ( 1 and 2$)$ were found intraoperative DSA in Hybrid-OR.

Furthermore, balloons could be directly deployed across aneurysms neck and block the blood for wide-necked carotid-ophthalmic aneurysms. This kind of neck across deployment was effective to decrease escape of blood clots in the aneurysm sac, proper placement of the aneurysm clip and good reconstruction of ICA. In our study, the balloon was deployed across the aneurysm neck in 5 cases with wide-neck ( $\geq 8 \mathrm{~mm}$ ). Four cases ( $80 \%$ ) got good reconstruction of the parent ICA, however, good reconstruction of the parent ICA could not be obtained in one case (Case 2) because of severe atherosclerosis in the aneurysm neck. no balloon-deployment related vasospasms occurred in the group of 12 patients.

\section{Conclusion}

In summary, microsurgical clipping, intraoperative ICG, and intraoperative monitoring techniques continued improving and progressing, and clipping of complex intracranial aneurysms is becoming increasingly safer. In accordance, balloons, stents and flow diverters make the treatment more and more familiar. However, there are still plenty of limitations and technical bottlenecks when these two types of methods are used individually. As Hybrid-OR comes to light, it provides a safer and more promising treatment platform for neurosurgery, providing a completely new concept for the treatment of intracranial aneurysms. Currently, our neurosurgery department has gained initial experiences. In our experience, Hybrid-OR is useful to greatly reduces the rate of incompletion of aneurysm clippings and vessel stenosis, and to preserve the important perforator vessels. Neurosurgeons can safely clip aneurysms and to the greatest extent to get ICA reconstruction in the Hybrid-OR, rather than tripping ICA and EC-IC bypass.

\section{Abbreviations}

CCA:common carotid artery; ICA = internal carotid artery; $\mathrm{MCA}=$ middle cerebral artery; $\mathrm{ACP}=$ anterior clinoid process; $\mathrm{AChoA}=$ anterior choroidal artery; PComA = posterior communicating artery, Hybrid-OR = Hybrid Operating Rooms; EC-IC = extracranial-intracranial; $I A C=$ intradural anterior clinoidectomy; EAC = extradural anterior clinoidectomy; DSA = digital subtraction angiography; $\mathrm{D} / \mathrm{N}=$ dome-neck; $\mathrm{mRS}=$ Modified Rankin Scale, $\mathrm{DDR}=$ distal dural ring; $\mathrm{DAPT}=$ dual antiplatelet therapy; SF = stent fracture, ISR = in-stent restenosis.

\section{Declarations}

\section{Ethics approval and consent to participate}

The studies involving human participants were reviewed and approved by Tianjin Medical University General Hospital ethics committee, the patients/participants provided their written informed consent to participate in this study.

\section{Consent for publication}

Written informed consents for the publication of personal data and images were obtained from all patients.

\section{Availability of data and materials}

The datasets used and/or analyzed during the current study are available from the corresponding author on reasonable request.

\section{Competing interests}

The authors declare that they have no competing interests.

\section{Funding}

No funding or any financial support was received in our study.

\section{Authors' contributions}

WQX and NZ designed the study, acquired the data and drafted the article, analyzed and interpreted the data, revised the article critically for important intellectual content together. All the authors approved the version to be published.

\section{Acknowledgements}

None.

\section{References}


1. Choudhri O, Mukerji N, Steinberg GK. Combined endovascular and microsurgical management of complex cerebral aneurysms. Frontiers in neurology 4(undefined), 108 (2013).

2. Kim SH, Song S, Kim SP et al. Hybrid technique to correct cerebral malperfusion following repair of a type a aortic dissection. The Korean journal of thoracic and cardiovascular surgery 47(2), 163-166 (2014).

3. Lloyd CT, Calafiore AM, Wilde P et al. Integrated left anterior small thoracotomy and angioplasty for coronary artery revascularization. The Annals of thoracic surgery 68(3), 908-911; discussion 911-902 (1999).

4. Ryttlefors M, Enblad P, Kerr RS, Molyneux AJ. International subarachnoid aneurysm trial of neurosurgical clipping versus endovascular coiling: subgroup analysis of 278 elderly patients. Stroke 39(10), 2720-2726 (2008).

5. Cockroft KM, Marks MP, Steinberg GK. Planned direct dual-modality treatment of complex broad-necked intracranial aneurysms: four technical case reports. Neurosurgery 46(1), 226-230; discussion 230-221 (2000).

6. Katz JM, Gologorsky Y, Tsiouris AJ et al. Is routine intraoperative angiography in the surgical treatment of cerebral aneurysms justified? A consecutive series of 147 aneurysms. Neurosurgery 58(4), 719-727; discussion 719-727 (2006).

7. Leung GK, Tsang AC, Lui WM. Pipeline embolization device for intracranial aneurysm: a systematic review. Clinical neuroradiology 22(4), 295-303 (2012).

8. Shapiro M, Becske T, Sahlein D, Babb J, Nelson PK. Stent-supported aneurysm coiling: a literature survey of treatment and follow-up. AJNR. American journal of neuroradiology 33(1), 159-163 (2012).

9. Day AL. Aneurysms of the ophthalmic segment. A clinical and anatomical analysis. Journal of neurosurgery 72(5), 677-691 (1990).

10. Tawk RG, Villalobos HJ, Levy El, Hopkins LN. Surgical decompression and coil removal for the recovery of vision after coiling and proximal occlusion of a clinoidal segment aneurysm: technical case report. Neurosurgery 58(6), E1217; discussion E1217 (2006).

11. Schmidt GW, Oster SF, Golnik KC et al. Isolated progressive visual loss after coiling of paraclinoid aneurysms. AJNR. American journal of neuroradiology 28(10), 1882-1889 (2007).

12. Park HK, Horowitz M, Jungreis $C$ et al. Endovascular treatment of paraclinoid aneurysms: experience with 73 patients. Neurosurgery 53(1), 14-23; discussion 24 (2003).

13. Khan N, Yoshimura S, Roth $\mathrm{P}$ et al. Conventional microsurgical treatment of paraclinoid aneurysms: state of the art with the use of the selective extradural anterior clinoidectomy SEAC. Acta neurochirurgica. Supplement 94(undefined), 23-29 (2005).

14. Sherif C, Gruber A, Dorfer C et al. Ruptured carotid artery aneurysms of the ophthalmic (C6) segment: clinical and angiographic long term follow-up of a multidisciplinary management strategy. Journal of neurology, neurosurgery, and psychiatry 80(11), 1261-1267 (2009).

15. Roy D, Raymond J, Bouthillier A et al. Endovascular treatment of ophthalmic segment aneurysms with Guglielmi detachable coils. AJNR. American journal of neuroradiology 18(7), 1207-1215 (1997).

16. Ng PY, Huddle D, Gunel M, Awad IA. Intraoperative endovascular treatment as an adjunct to microsurgical clipping of paraclinoid aneurysms. Journal of neurosurgery 93(4), 554-560 (2000).

\section{Figures}




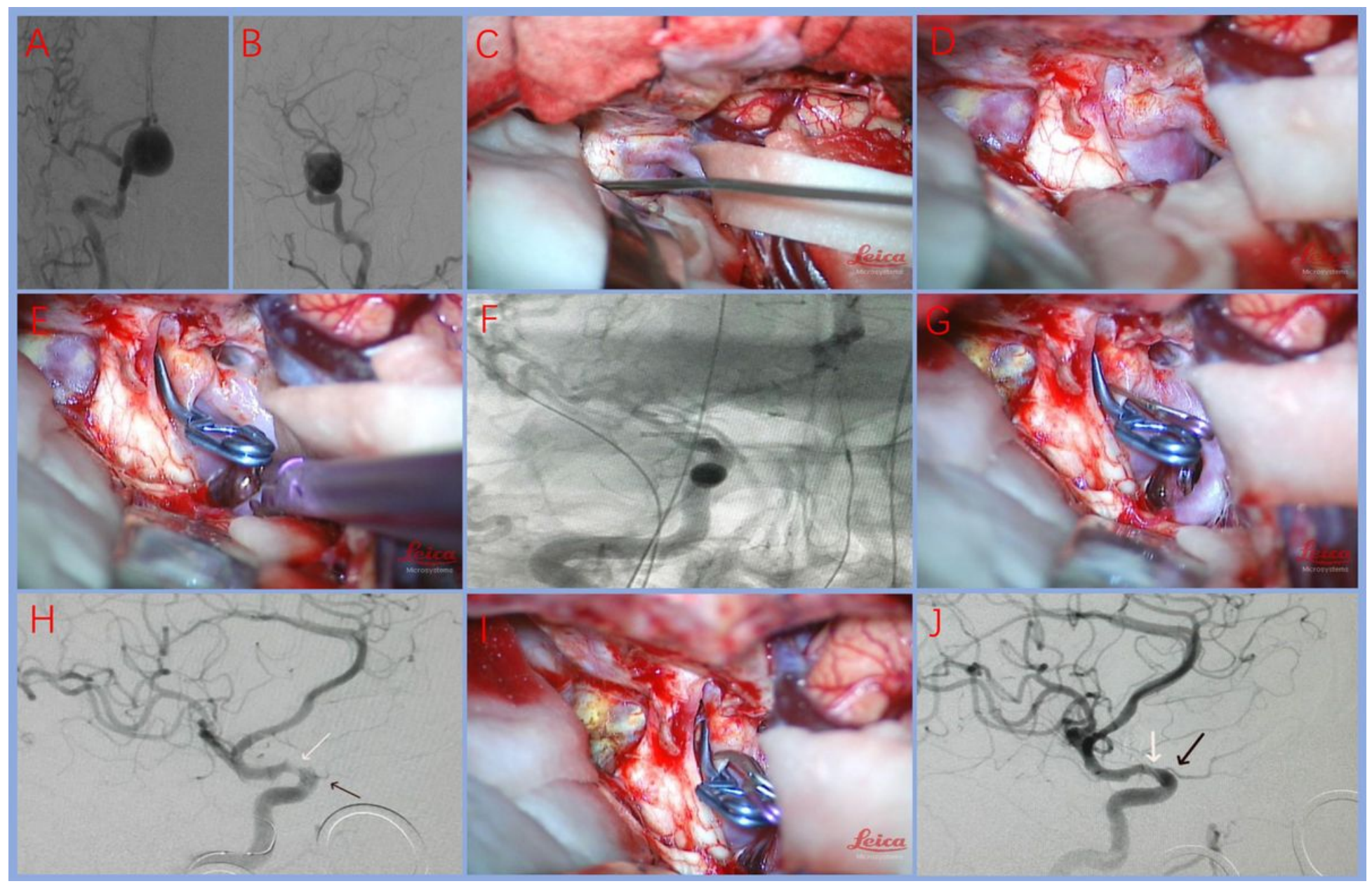

\section{Figure 1}

Figure1A\&B: Pre-operation DSA in AP \& LAT views: the stained aneurysm was visible in dextral ICA ophthalmic artery segment, Maximal diameter (25.3) mm, Neck size (7.5 mm), D/N ratio 3.4. Figure1C\&D: Showing the optic nerve was compressed by the giant aneurysm; Removal of the anterior clinoid process and dissociation of the optic nerve from the aneurysm neck to sufficiently reveal the aneurysm neck. Figure1E\&F: Placement of the first clip, the first intraoperative DSA displayed that the aneurysm has been clipped囚but a small portion of the aneurysm neck was residue and visualized. Figure1G\&H: Supplementary miniature clip was placed during operation; the second intraoperative DSA displays that the aneurysm has been completely clipped, however, stenosis occurred in the ICA (white arrow) with poor visualization of the optic nerve (black arrow) and poor visualization of the distal branch vessel of MCA. Figure1 \& J: A miniature clip with angular tips was used to replace the original one. The third intraoperative DSA displayed that: the aneurysm was completely clipped. The ICA stenosis and the ophthalmic artery stenosis relieved (white arrow), and distal branch vessel of MCA was effectively improved (black arrow). 


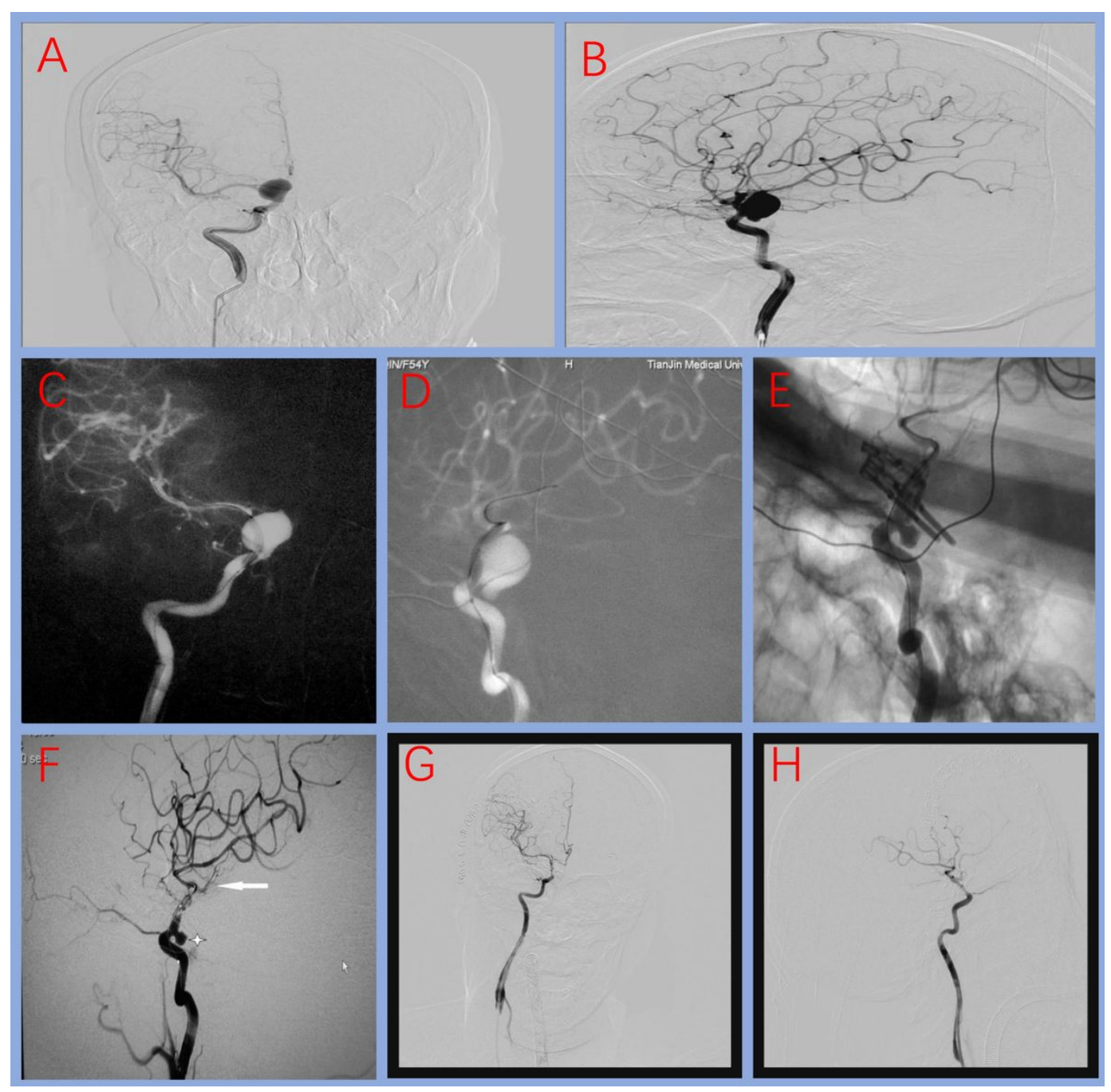

\section{Figure 2}

Figure2A\&B: Pre-operative DSA in AP \& LAT views: the giant stained aneurysm in the internal carotid-ophthalmic segment was visible, maximal diameter (20.4 $\mathrm{mm}$ ), neck size (13.4 mm) D/N ratio 1.6. Figure2C\&D: The first intraoperative DSA (AP \& LAT view) displayed the balloon were placed in the aneurysm neck. Figure2E\&F: The second intraoperative DSA displayed that the bulk of aneurysm was clipped and not visualized, but a small portion of the aneurysm was residue and visualized. Figure2G\&H: After adjusting clips, the third intraoperative DSA displayed the aneurysm was completely clipped, but the parent artery is slightly narrowed due to the severe calcification of the aneurysmal neck. 